\title{
Beiträge zur Physiologie des Sehens. III.
}

\author{
Von
}

C. Baumann.

(Mit 3 Textfiguren.)

Bei dem in Bd. 95 S. 364 dieser Zeitschrift angegebenen Versuche mit dem Stereoskope sieht das eine Auge durch ein rothes Glas; während das andere Ange gleichzeitig durch ein blaues Glas hindurchsieht. Beide Gläser sind mit einem ca. $1 \mathrm{~mm}$ breiten offenen Spalte versehen; der Spalt deś einen Glases läuft senkrecht, der des anderen Glases waagerecht. Der Versuch zeigt deutlich, dass die Wahrnehmung des rothsehenden Auges durch den Gesichtssinn in eine andere Ebene verlegt. wird als die Wabrnehmung des blausehenden Auges, weil wir, durch den freien Zwischenraum im rothen Glase hindurchsehend, das blaue Bild sehen können. Es muss also eine ungleiche Accomodation der beiden Augen im Momente der Wahrnehmung stattgefunden haben.

Wenn diese Folgerung richtig ist, so muss sich weiter erweisen lassen, dass ein für Roth accommodirtes Auge eine andere Farbe nicht gleichzeitig scharf zu sehen vermag, wenn beide Farben sich in der gleichen Entfernung rom Auge befinden. Die folgenden Versuche haben nun diese Folgerung bestätigt, indem dieselben zeigen, dass zwẹi abweichende Farben sich in ungleichen Entfernungen vom Auge befinden müssen, um eine gleichzeitige scharfe Wahrnehmung zu ermöglichen. Die gefundenen Entfernungsunterschiede sind auch geeignet, einen Maassstab zu bilden für die Arbeitsleistung des Auges bei der Accommodation.

Zu dem Versuche dient ein cylindrischer Kork $K$, welcher mit einer entsprechend langen Nadel centrisch durchbohrt ist, wie dies in der umstehenden Fig. 1 angedeutet ist. Die Nadel lässt sich mit Hülfe des an ihrem unteren Ende befindlichen Knopfes leicht hin und her schieben. Während sich auf der oberen Korkfläche ein rundes Stück rothen Papieres von $25 \mathrm{~mm}$ Durchmesser befindet, 
bringt man auf der Nadelspitze ein dünnes Korkscheibchen mit farbigem Papiere von $10 \mathrm{~mm}$ Durchmesser an. Beim Betrachten von oben nimmt man eine kleine Scheibe farbigen Papieres inmitten einer grösseren rothen Fläche wahr, wie in Fig. 2 angedeutet ist. Da die Fläche, welche uns in einer Einzelwahrnehmung scharf erscheint, sehr klein ist, so ist es nöthig, zur Vergleichung der Schärfe den Blick fest auf einen bestimmten Punkt zu richten, und desshalb sind auf dem kleineren Scheibchen wie auch auf der grossen Scheibe mit $K$ weisser oder schwarzer Farbe kleine, scharf begrenzte Punkte aufgezeichnet. Beim Beobachten wird die kleine Scheibe so gehalten und gedreht, dass die beiden Punkte unmittelbar neben einander za liegen scheinen, damit die unmittelbare Umgebung der Punkte in Bezug auf ihre Schärfe genau beobachtet und verglichen werden kann. Um auch die Sehweite

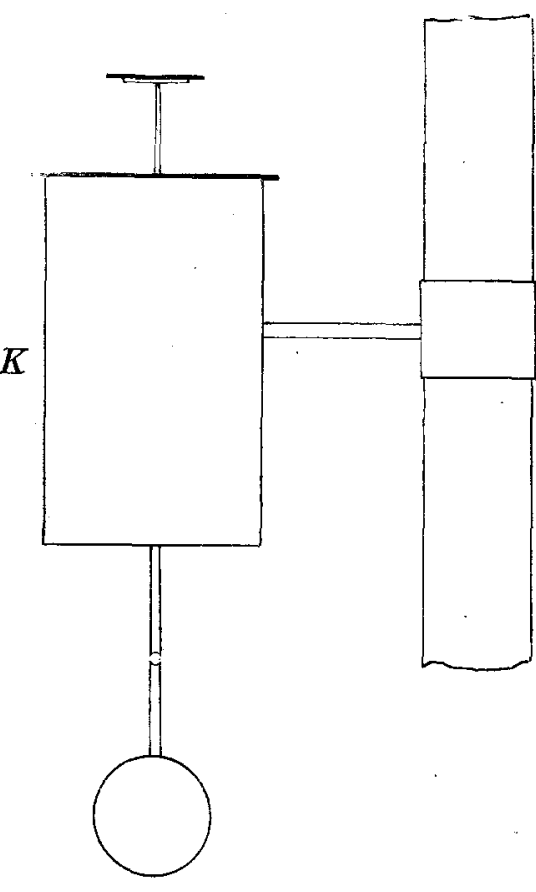

Fig. 1.

des beobachtenden Auges feststellen zu können, wird der Kork mittelst eines Drahtes, welcher an einer Schlaufe befestigt ist, mit einem graduirten Stabe verbunden.

Die Beobachtungen selbst sind für die Augen in hohem

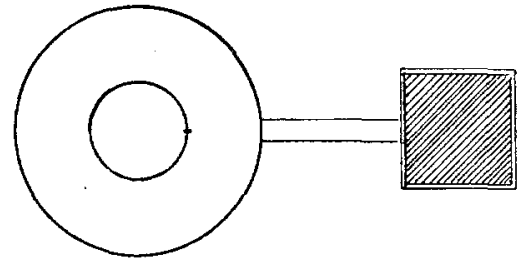

Fig. 2. Grade anstrengend und dürfen nicht bis zur Ermüdung derselben getrieben werden, weil dann die Wahrnehmungen undeutlich und unzuverlässig werden. Es ist weiter zu berücksichtigen, dass die Tiefenschärfe nicht bloss auf einen mathematischen Punkt beschränkt ist, sondern einen kleinen Spielraum hat, welcher für mein Auge etwa $3 \mathrm{~mm}$ beträgt; in Folge dessen vermag ich zwei Punkte von zwei gleich- 
farbigen Scheiben noch scharf zu sehen, welche $3 \mathrm{~mm}$ von einander entfernt sind. Am zuverlässigsten scheinen die monocularen Beobachtungen zu sein ohne Benutzung von Augengläsern, welche leicht störende Reflexbilder verursachen.

Die in Folge von Beobachtungen sich leicht und bald einstellenden complementär gefärbten Nachbilder machen die Wahrnehmungen ganz unbrauchbar. Um den Augen Gelegenheit zur Erholung zu geben, ist das Belegen des Arbeitstisches mit schwarzem Stoffe oder Papier nothwendig, und um zu wissen, ob das beobachtende Auge völlig ausgeruht ist, überzeuge man sich, ob ein Stück weissen Papieres beim abwechselnden Betrachten mit dem beobachtenden und dem nicht beobachtenden Auge beiden Augen gleichfarbig erscheint. Ohne Anwendung dieser Vorsichtsmaassregeln ergaben sich unbrauchbare Resultate.

Für mein rechtes Auge ergaben sich am 29. März 1904 bei bedecktem Himmel folgende Entfernungsunterschiede:

$\begin{array}{cccc}\text { für } & \text { für } & \text { für } & \text { für } \\ \text { Gelb } & \text { Hellgrün } & \text { Dunkelgrün } & \text { Blau } \\ 9 & 17 & 19 & 25\end{array}$

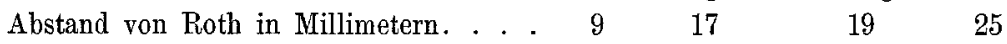

Da die Sehweite $26,5 \mathrm{~cm}$ beträgt, so er-

gibt dies in Procenten ausgedrückt . . 3,4\% $\%, 4 \% \quad 7,17 \% \quad 9,44 \%$

Es sind das so grosse Zahlen, dass wir uns erstaunt fragen, wie es kommt, dass uns dieses Verhalten der Farben zu einander nur in verhältnissmässig seltenen Fällen und nicht fortwährend stört. Nun sind wir aber von frühester Jugend an gewohnt, die Augen umherschweifen zu lassen, und darum ergibt sich für unseren Gesichtssinn selten eine Gelegenheit, darauf aufmerksam zu werden. Für gewöhnlich lenkt uns also die fortwährende Aenderung der Blickrichtung von der Wahrnehmung ab, und bei scharfer Beobachtung behindert uns bald die Ermüdung der Augen, das Eintreten der complementär gefärbten Nachbilder, wie dies die vorhin angegebenen Vorsichtsmaassregeln darthun, welche für eine zuverlässige Beobachtung nothwendig sind. Vor der Anwendung jener Vorsichtsmaassregeln meinerseits waren die Ergebnisse dermaassen schwankend und unsicher, dass sie ganz unbrauchbar waren. Hierbei zeigte sich, dass die Entfernung der beiden Scheiben in dem Maasse abnahm, in welchem die Ermüdung der Augen zunahm.' Es trat als Folge der Frmüdung ein Nachlassen der Ciliarmuskelthätigkeit und dadurch eine Verminderung der Accommodation ein, welche ihrerseits dann die Minderung der Schärfe des Bildes bewirkte. 
Soll ein rother Lichtstrahl ebenso stark gebrochen werden wie ein blauer Strahl, so kann dies nur geschehen, wenn für Roth ein stärker brechendes Medium in Anwendung kommt als für Blau, und dieser Grund war bestimmend für die Natur, beim Auge das Brechungsvermögen der Linse veränderlich zu gestalten. In der That wird das Brechungsvermögen der Linse in Folge der Accomodation durch den Reiz, welchen die Strahlen verschiedener Wellenlängen in verschieden starker Weise auf das Auge ausüben, abgeändert (v. Helmholtz, Handbuch der physiol. Optik $\S 12$ S. 130-156). Dass die Accommodation für die Strahlen verschiedener Wellenlängen auch versehieden stark sein muss, ergibt sich als ein Gebot der Nothwendigkeit.

Die obigen Versuche sind mit käuflichem Papier gemacht worden; voraussichtlich werden daher anderweitige Versuche etwas andere Ergebnisse haben. Immerhin bieten die erhaltenen Zahlen schon einen Anhalt für die verschieden starke Arbeitsleistung des Auges bei der Accommodation. Wenn es gelänge, diese Versuche mit Spcetralfarben auszuführen, so würde sich genau ermitteln

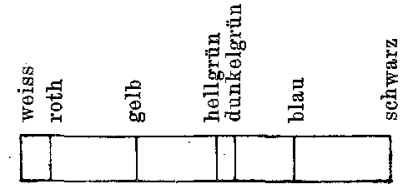

Fig. 3. lassen, welcher Grad von Accommodation durch die Strahlen einer bestimmten Wellenlänge herbeigeführt wird. In Figur 3 sind die erhaltenen Maasse eingezeichnet und auch eine Beobachtung mit schwarzem sowie mit weissem Papier zugefügt. Die Beobachtungen mit weissem Papiere ergaben bei bewölktem Himmel und mittelstarkem Lichte eine $3 \mathrm{~mm}$ stärkere Accommodation als die durch Roth hervorgerufene; wahrscheinlich ergeben sich bei stärkerem Lichte noch grössere Abstände für weisses Papier.

Bei der Einstellung für die Nähe, also auch für die Farben ist die Wirkung der Iris darauf berechnet, die seitlich einfallenden Strahlen, welche nicht zur Vereinigung gebracht werden können und die Klarheit des Bildes störend beeinflussen würden, vom Auge fernzuhalten und dient mithin dazu, die Leistungsfähigkeit der Linse zu unterstützen. In Folge dessen wird die Netzhautfläche von den verschiedenartigen Strahlen ungleich stark in Anspruch genommen. Während die schwarzen und blauen Strahlen sich über die ganze Netzhaut verbreiten können, sind die anderen Strahlen je nach $\mathrm{Zu}$ nahme ihrer Wellenlängen darin beschränkt, am meisten natürlich 
die rothen Strahlen. Diese Beschränkung der rothen Strablen auf den centraleren Theil der Netzhaut gibt uns auch die Erklärung dafür, wesshalb diese bei gutem Lichte intensiver wirken als blaue. Wenn man nämlich im Stereoskope mit durchfallendem Lichte dem einen Auge ein blaues Glas vorfihhrt und dem anderen ein rothes, so kann man sich überzengen, dass bei dem Wettstreite der beiden Augen, ihre gesonderten Wahrnehmungen in dem gemeinschaftlichen Bilde zum Ausdrucke zu bringen, das rothe Bild zeitlich zwar kürzer zur Herrschaft gelangt, aber mit grösserer Intensität als das blaue Bild, während letzteres zeitlich länger überwiegt, dafür aber nicht mit der gleichen Intensität wie das rothe.

Die beobachtete Abnahme der lichtempfindlichen Elemente, Zäpfchen und Stäbchen in der Netzhaut von der Mitte nach den Rändern hin (siehe „Experimentelles über das Sehen im Dunkeln und Hellen" [ "Hypothese über die Ursache der Farbenblindheit"] von O. Lummer, vorgetragen am 22. Jan. 1904 in der Sitzung der Deutsehen physik. Gesellschaft, veröffentlicht in den. Verhandlungen d. D. physik. Ges. von KarI Scheel, 6. Jahrgang Nr. 2, S. 7-77. 30. Jan. 1904, Braunschweig, Druck und Verlag von Friedr. Vieweg \& Sohn, 1904), lässt die Erklärung zu, dass die Abnahme dieser Elemente eine verminderte Wirkung auf die Netzhaut herbeifubhren müsse. Es kann aber auch gefolgert werden, dass in Folge der veränderten Inanspruchnahme der Thätigkeit der Netzhaut die Reizbarkeit für die längeren Lichtstrahlen bei jenen Elementen, welche in den weniger centralen Theilen der Netzhaut gelagert sind, abgenommen habe.

Stützt man sich auf die Beobachtung, dass bei unseren gebräuchlichen Objectiven die Helligkeit des Bildes in der Camera von der Mitte aus nach den Rändern hin abnimmt, so dürfen wir für die Linse im Auge ein gleiches Verhalten voraussetzen. Die verminderte Lichtstärke der aussercentralen Strahlen bedingt von selbst eine verminderte Wirkung auf die äusseren Netzhauttheile, und es ist daher folgerichtig anzunehmen, dass die stärkere Wirkung auf dem centralen Theile der Netzhaut zum Ausdruck kommen muss. Dies ist ein weiterer Grund für die Wahrscheinlichkeit, dass die Leuchtkraft von Roth bei starikem Lichte in ursächlichem Zusammenhange steht mit der Beschränkung der rotben Strahlen auf den centraleren Theil der Netzhaut, ebenso wie die Sichtbarkeit des Blau selbst bei schwachem Lichte mit der Einwirkung auf eine weit grössere Netzhautfläche zu erklären ist. 
Beim Arbeiten in einem Raume, welcher nur durch rothes Licht erhellt ist, hat die stärkere Reizung des Auges den Uebelstand, dass man die Entfernung der Körper zunächst unterschätzt. Hierdurch wird man in der Abschätzung der Entfernungen urisicher gemacht; bestrebt, sich zu verbessern, greift man alsdann zu weit, und es dauert eine Weile, bis man lernt, die Entfernungen richtig $\mathrm{zu}$ schätzen und auch richtig zu greifen. Der Uebergang aus dem rothen Lichte in's weisse Licht ist für die Augen unangenehmer als der Uebergang aus gelbem Lichte in weisses Tageslicht. - Das Verhalten der Menschen und mancher Thiere gegen plötzlich auftretendes Roth haben wir im „II. Beitrage zur Physiologie des Sehens" (Band 95 S. 366) bereits besprochen. Die oben mitgeteilten Versuchsergebnisse stehen mit der früheren Erklärung über die Rothwirkung im Einklange. Es ist ferner wahrscheinlich, dass das Eintreten von Gesichtsschwindel im Zusammenhange steht mit einer raschen Abänderung der Accommodation des Auges.

Die begeisterte Erregung, welche sich der Franzosen bemächtigt, wenn sie ihre Truppen auf dem Paradefelde bei schönem Wetter, d. h. bei starkem Lichte, manövriren sehen, bängt zum grossen Theile mit der Wirkung des Roth auf die Augen der Zuschauer zusammen. Es ist jedoch unrichtig, aus dieser Wirkung zu schliessen, der rothbekleidete Soldat erhalte ein besonders "martialisches" Aussehen, wie die Franzosen uns glauben machen wollen. Zudem ist kein Beispiel bekannt, aus welchem hervorgeht, dass jenes martialische Aussehen den rothbekleideten Soldaten in einem höheren Maasse zukomme als den nicht rotbbekleideten Soldaten. Unsere Erfahrung gestattet uns jedoch, zu behaupten, dass die Erregung der Aufmerksamkeit feindlicher Augen. im Kriegsfalle für die Träger rother Kleidungsstücke viel häufiger schädlich als nützlich war. 